\section{INCONTINENCIA URINARIA}

\author{
Dr. Pablo Gómez Martinez*
}

Dr. Alvaro Fonnegra Miramón**

Dr. Jaime Cantillo Guerrero***

La incontinencia urinaria ha sido desde hace tiempo un problema difícil de resolver y un desafío tanto para el ginecólogo como para el urólogo, por los variados conceptos en su fisiopatología, muchos de ellos contradictorios, y por los resultados inconstantes que se obtienen con la cirugía.

La existencia del esfínter propiamente dicho del cuello vesical, -no se acepta- hoy en día, y como veremos luego, la continencia de la orina se debe principalmente a un complejo mecanismo muscular que se extiende desde uréter, trígono vesical, hasta la porción terminal de la uretra y en el cual las fibras musculares que forman y envuelven a esta última, son una misma estructura descle el punto de vista embriológico, anatómico y fisiológico.

\section{Consideraciones embriológicas:}

Cuando la cloaca se abre por primera vez al exterior, el seno urogenital es una estructura continua con la alantoides. En este estado, se puede dividir en una porción ventral o pélvica que viene a formar la vejiga y una porción uretral que recibe los conductos mesonéfricos y los canales de Müller fusionados, y que vie- ne a constituir la uretra prostática y membranosa en el hombre, y la totalidad de la uretra y el vestíbulo en la mujer.

Es claro pues, que el músculo vesical (detrusor) y la musculatura uretral tienen el mismo origen y son una estructura continua que se puede asimilar a un tubo con una dilatación superior.

\section{Consideraciones anatómicas e histológicas:}

Además de la continuidad que se acaba de mencionar, se encuentra una distribución muy particular de las fibras musculares que unen la vejiga y la uretra.

Para comprobar estos hechos, enunciados en 1968 por Tanagho, Meyers y Smith (1), (2), hicimos la disección del trígono vesical separándose muy bien sus dos capas como puede verse en la Fig. No 1. Tam-

* Profesor de Urología de la Facultad de Medicina de la Universidad Nacional de Colombia.

** Profesor Asociado de Ginecología de la Facultad de Medicina de la Universidad Nacional de Colombia.

*** Instructor Asociado de Anatomía Patológica de la Facultad de Medicina de la Universidad Nacional de Colombia. 


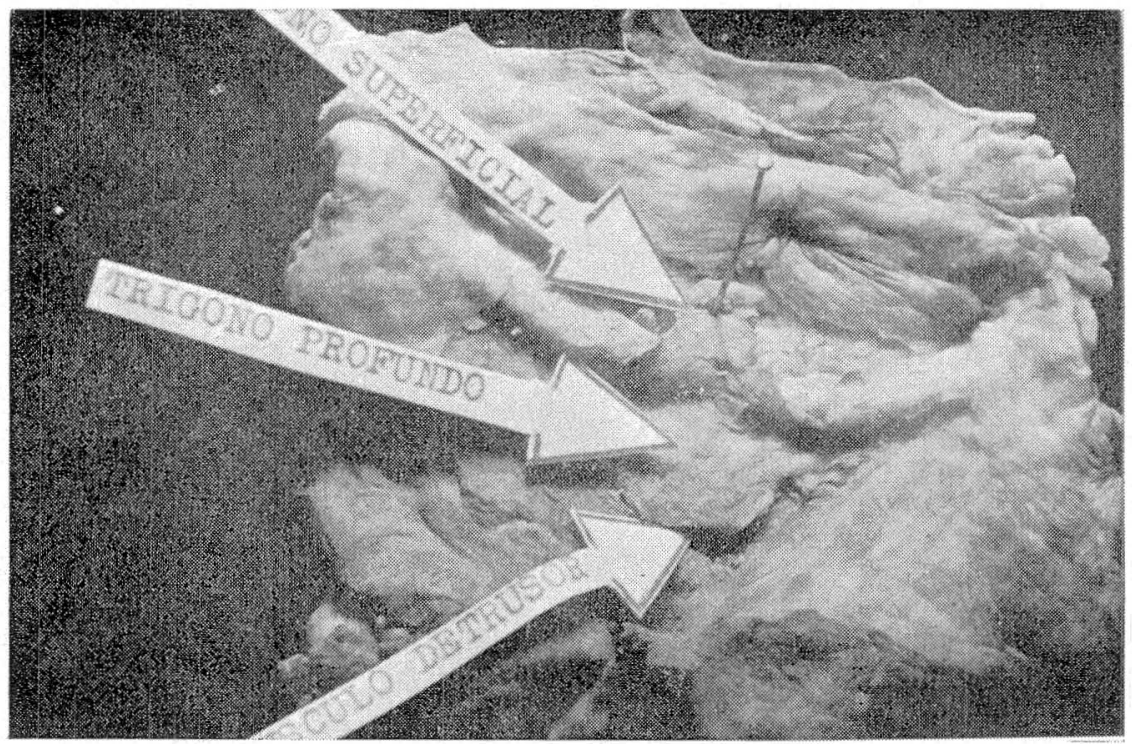

FIGURA № 1 - Esta fotografía muestra el trígono superficial, el profundo y el músculo detrusor de la vejiga.

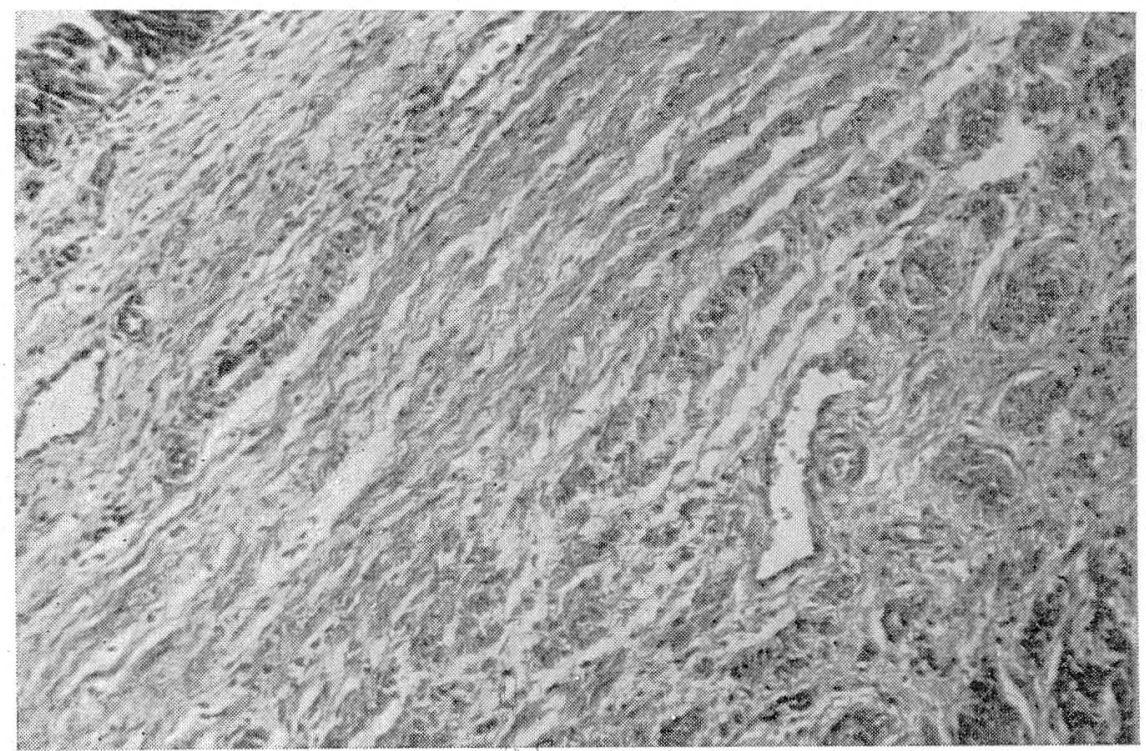

FIGURA No 2 - En el ángulo superior izquierdo se ve la mucosa vesical con su epitelio de transición y hacia abajo las capas del trígono y el músculo detrusor. 
bién practicamos cortes histológicos coloreándolos con hematoxilina eosina comprobándose lo anteriormente dicho (Fig. № 2 ).

De acuerdo con lo comprobado anteriormente, el trígono superficial continúa directamente al uréter hacia abajo (Fig. № 3) y el trígono profundo lo hace con la lámina o envoltura de Waldeyers, (Fig. No 4), de tal suerte que este último sirve de eslabón entre las estructuras mesodérmicas del uréter y las endodérmicas del cuello de la vejiga.

Por otra parte el músculo detrusor, continuación directa del longitudinal de la vejiga, con sus tres clases de fibras : longitudinales internas, externas y circulares medias, son fundamentales en el mecanismo de continencia urinaria, pues las dos primeras forman la capa longitudinal interna y la circular externa de la uretra, que junto con la circular media y el trígono, forman el mecanismo cle cierre del cuello vesical y la continencia uretral.

Es claro que el mecanismo esfinteriano no está localizado en un solo nivel, sino que forma largo segmento que rodea la uretra en sus $3 / 4$ partes proximales, cuya contracción es la que produce un aumento de la presión intrauretral, tanto más alta, cuanto mayor sea la acción de las fibras en arco y mayor su número a lo largo del canal.

\section{Consideraciones fisiopatogénicas:}

De los hechos anatómicos enunciados se deduce que la continencia de la orina es la resultante de la resistencia que opone el mecanismo esfinteriano a la presión endovesical. Debe recordarse que una de las características del detrusor vesical es

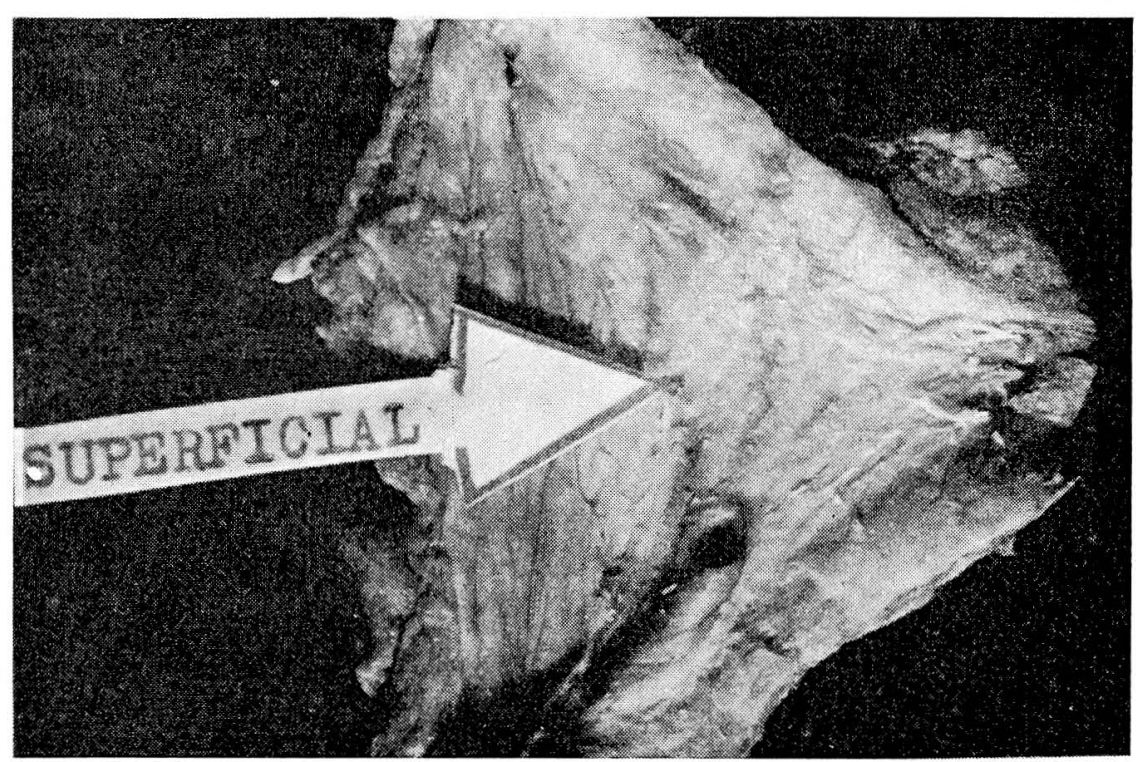

FIGURA No 3 - La fotografía presenta el uréter que se pierde insensiblemente para formar el trígono superficial al encontrarse con el del otro lado. Este hecho se logra ver quitando la mucosa. 


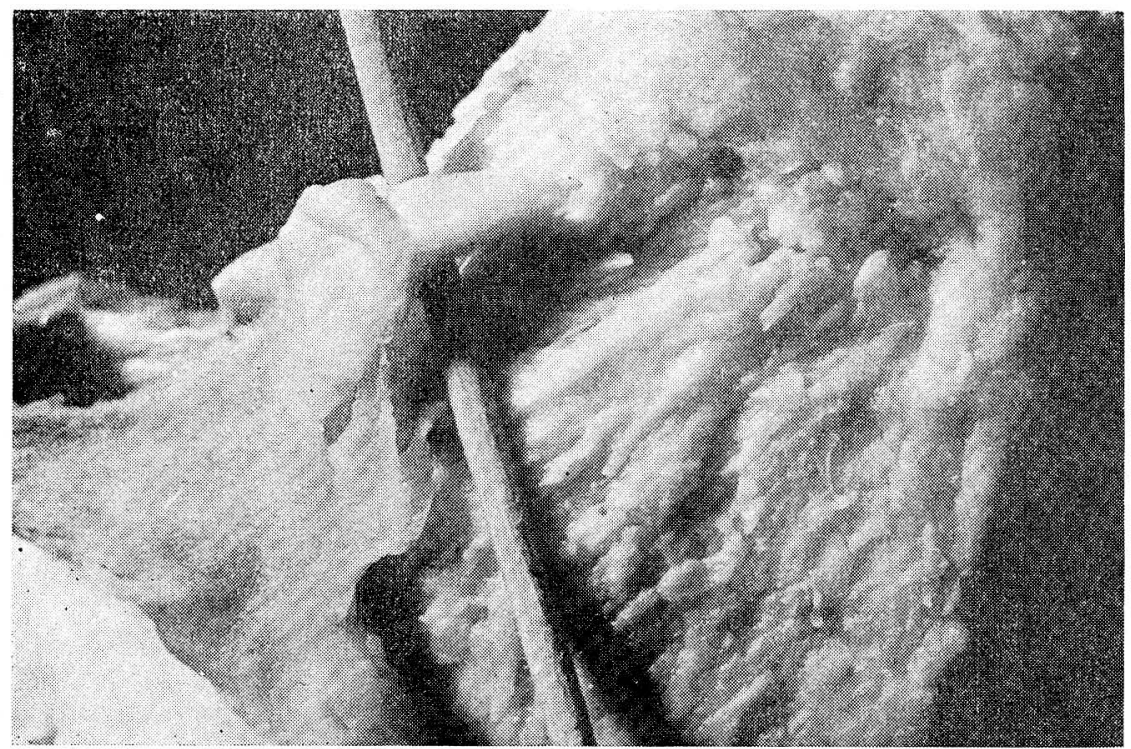

FIGURA No 4 - El uréter abrazado por la lámina de Waldeyer, la cual forma el trígono profundo.

la capacidad de adaptarse a contenidos variables conservando una presión baja. Ahora bien, la salida de orina involuntaria (incontinencia) se presenta en sólo dos circunstancias: - cuando la presión intravesical es mayor que la resistencia que le opohe el mecanismo esfinteriano, o cuando la presión que ejerce éste, en las paredes uretrales está disminuída y ès menor que la presión vesical, aún siendo ésta normal, (3).

Son muchas las consideraciones que se pueden hacer sobre las alteraciones patológicas que determinan en la mujer la incontinencia, particularmente la de esfuerzo.

El piso pélvico, incluyendo el diagrama urogenital, tiene dos curvas: una en dirección antero-posterior, la otra en sentido lateral. Tiene un grado de concavidad hacia la cavidad pélvica. Cuando se contrae, este gra- do de concavidad disminuye y el contenido pélvico es empujado hacia arriba. Cuando se relaja, tanto él, como el contenido pélvico, caen hacia abajo. Al estado de reposo, el piso pélvico permanece entre estos dos extremos.

La uretra bien acoplada al diafragma urogenital, que forma parte del piso pélvico sigue cualquier cambio que se produzca en ese sitio. En posición de descanso, el segmento vesical alrededor del meato interno es plano y mantiene un cierto ángulo con la uretra. En relación con la pelvis (como al iniciarse el vaciamiento), el piso pélvico se va hacia abajo, llevándose con él a la uretra, que necesariamente empuja sobre la base de la vejiga. Esto da por resultado que la forma plana de la base vesical se pierde y el ángulo uretro-vesical se altere, conduciendo al debilitamiento de las fibras circulares o en 
cabestrillo, que se envuelven alrededor de la uretra. Así se crea una situación que altera el mecanismo de cierre de la presión uretral. Por esta razón, la relajación pélvica, usualmente ocurre algunos segundos antes que el detrusor se contraiga y el vaciamiento comience. Sin embargo, si el relajamiento pélvico no es voluntario, sino el resultado de una permanente debilidad del piso pélvico, causado por repetida preñez, trabajo y traumas de partos, procesos operatorios, senilidad o desarrollo inadecuado, entonces estos cambios en la anatomía uretrovesical son también permanentes y la paciente tendrá la resistencia uretral a su más mínimo nivel, dando como resultado la incontinencia de esfuerzo. El mismo mecanismo de debilidad pélvica conduce frecuentemente a la formación de una hernia vesical o cistocele. A veces es favorable cuando la hernia corrige el ángulo uretro vesical, pero si lo supera, puede provocarse incontinencia, de ahí, que después de corrección de cistocele no es infrecuente que la paciente que antes tenía continencia, empiece a padecer de incontinencia en el post-operatorio inmediato. (4), (5), (6), (7).

\section{Principales tipos de incontinencia urinaria:}

Siguiendo este mismo orden de ideas, son muchas las variedades de incontinencia que se pueden presentar, cada una de las cuales debe tener un tratamiento distinto, de acuerdo con la patología presente, con el ánimo de restablecer la buena orientación de las fibras musculares para que ellas puedan desempeñar su papel, el ángulo uretro-vesical y el soporte del piso pélvico.

\section{Así tenemos:}

Incontinencia por infecciones severas, agudas y crónicas;

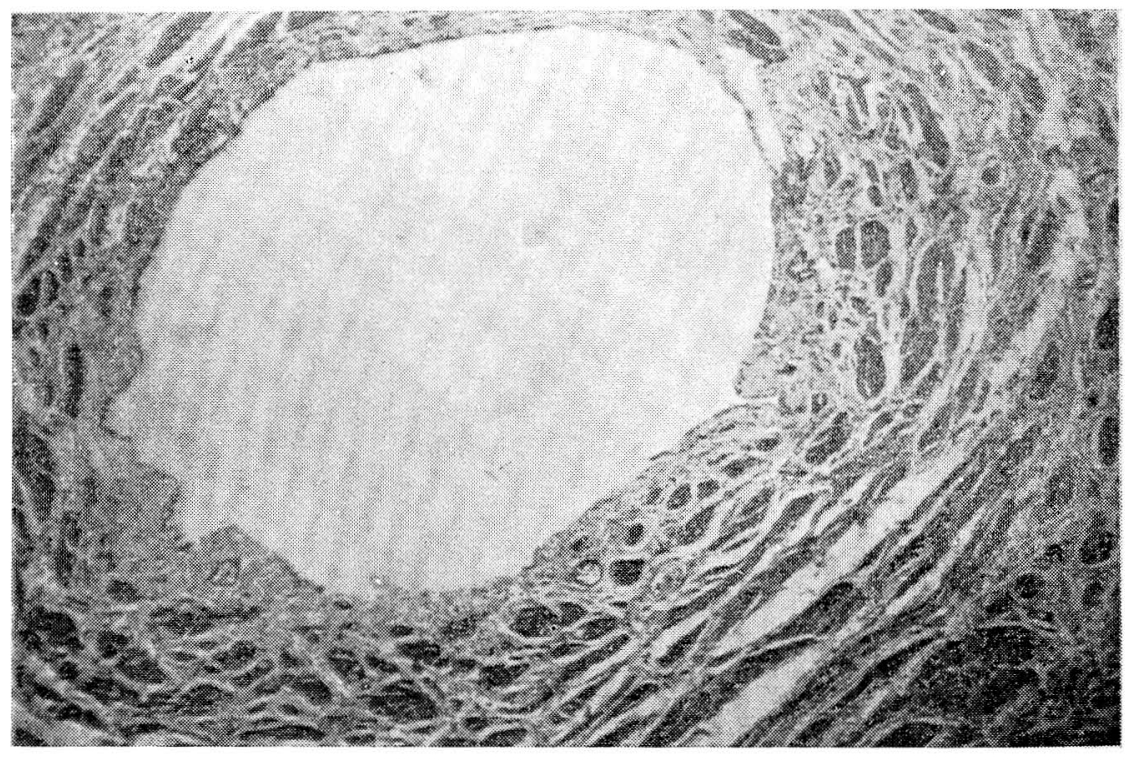

FIGURA $\mathrm{N}^{0} 5$ - Un corte del uréter en la mitad del espesor de la pared vesical, apreciándose cilíndíco, para aplanarse progresivamente, para evitar el reflujo. 


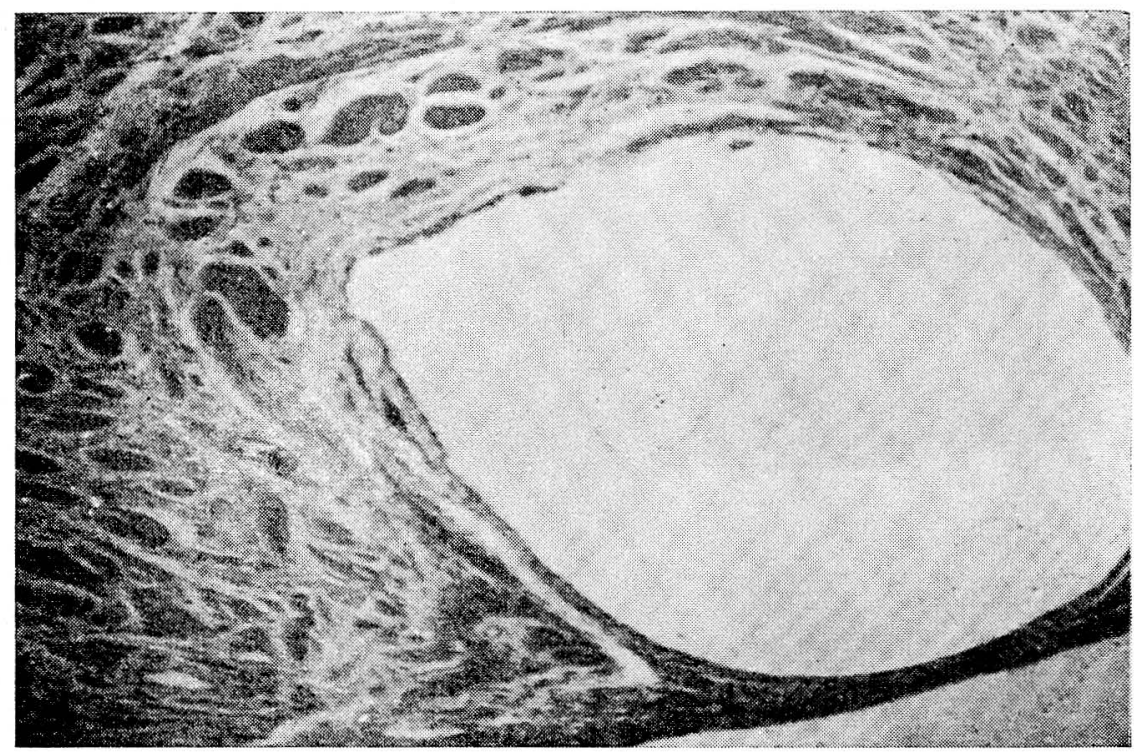

FIGURA No 6 - Un corte de uréter antes de desembocar en la vejiga viéndose aplanado, reduciendo su luz para evitar el reflujo.

Incontinencia de tipo congénito: en hipospadias, epispadias, defectos del diafragma uro-genital, dilatación congénita femenina, brevedad del canal;

Incontinencia de esfuerzo, la más común;

Incontinencia por alteración neurogénica;

Incontinencia por alteraciones psíquicas.

\section{Aspectos quirúrgicos:}

Las operaciones tipo Kelly, o Kennedy-Kelly para incontinencia urinaria de esfuerzo, se deben emplear cuando hay incontinencia con o sin cistocele, para corregir la pérdida del ángulo uretro-vesical y disminuir la dilatación uretral presente en menor - mayor grado, reforzando el mecanismo muscular del cuello de vejiga y uretra, siempre como paso previo a cirugía mayor de tipo abdominal. (Figs. Nos. 5, 6, 7 y 8 ).

La operación de Marshall-Marcheti debe ser seleccionada solamente para los casos en que fracase el procedimiento anterior; de la imposibilidad de operar por vía vaginal, como en el caso de las vírgenes; de la necesidad de practicar laparotomía por afección concomitante y del resultado satisfactorio de la prueba de Marshall (4).

En el grupo de incontinencia de tipo congénito, hemos hecho varias técnicas quirúrgicas; para la dilatación de la uretra: operación de Young o plastias en VY del cuello. Para las uretras cortas alargamiento de Leadbetter, con o sin reimplantación uretral. (Figs. Nos. 9, 10 y 11).

Para la dilatación congénita del cuello vesical: la resección y sutura 


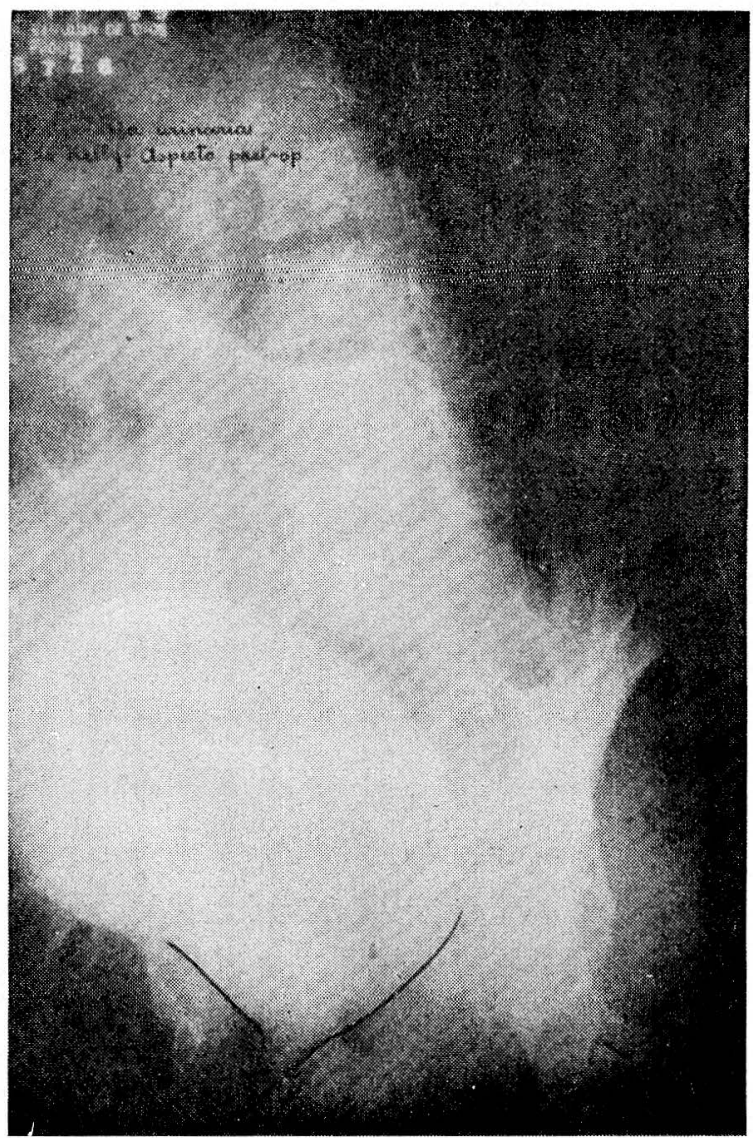

FIGURA No 7 - Aspecto radiológico preoperatorio, detalle de los ángulos y globo vesical (Operación de Kelly).

de Milling o la operación de Tanagho.

\section{Resumen y Conclusiones}

De acuerdo con las tesis expuestas y suficientemente probadas, sobre la continencia urinaria:

1. No existe esfínter propiamente dicho, sino un mecanismo esfinteriano compuesto por un complejo mecanismo muscular que se extiende desde el uréter, trígono vesical, cuello de vejiga y uretra;
2. La continencia urinaria depende de cuatro mecanismos principalmente:

a. Aparato urinario intrínseco bajo

b. Diafragma uro-genital

c. Presión intra-abdominal

d. Angulo uretro-vesical

3. Cualquier situación que rompa los mecanismos antes dichos, total o parcialmente provocará incontinencia urinaria; 


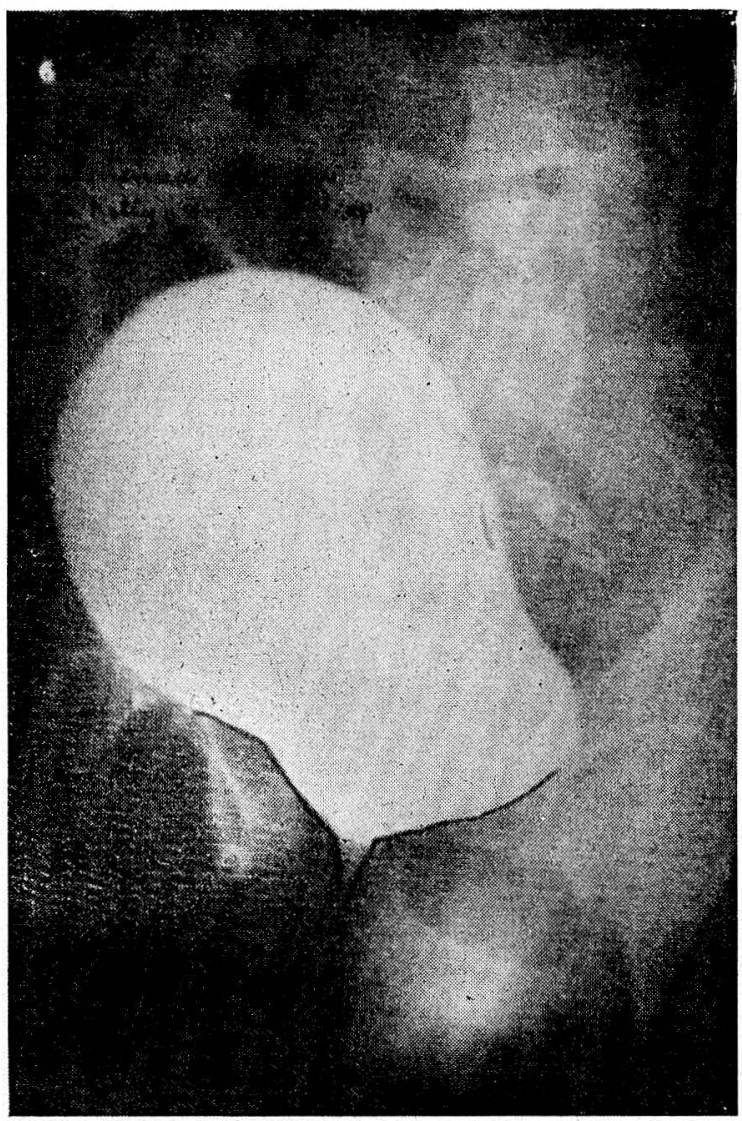

FIGURA № 8 - Aspecto radiológico post-operatorio (Operación de Kelly), detalles de los ángulos $y$ del globo vesical.

4. De acuerdo con lo anterior existen por consiguiente varios tipos de incontinencia urinaria;

5. Cada tipo de incontinencia urinaria, tiene su modalidad de corrección y por tanto, el diagnóstico debe ser lo más exacto posible, con el objeto de aplicar a cada caso, el tratamiento quirúrgico correspondiente;

6. Se mencionan algunas técnicas quirúrgicas para corregir diversos tipos de incontinencia, sin entrar en detalle de procedimiento. 
Vol. XXII

№ 4

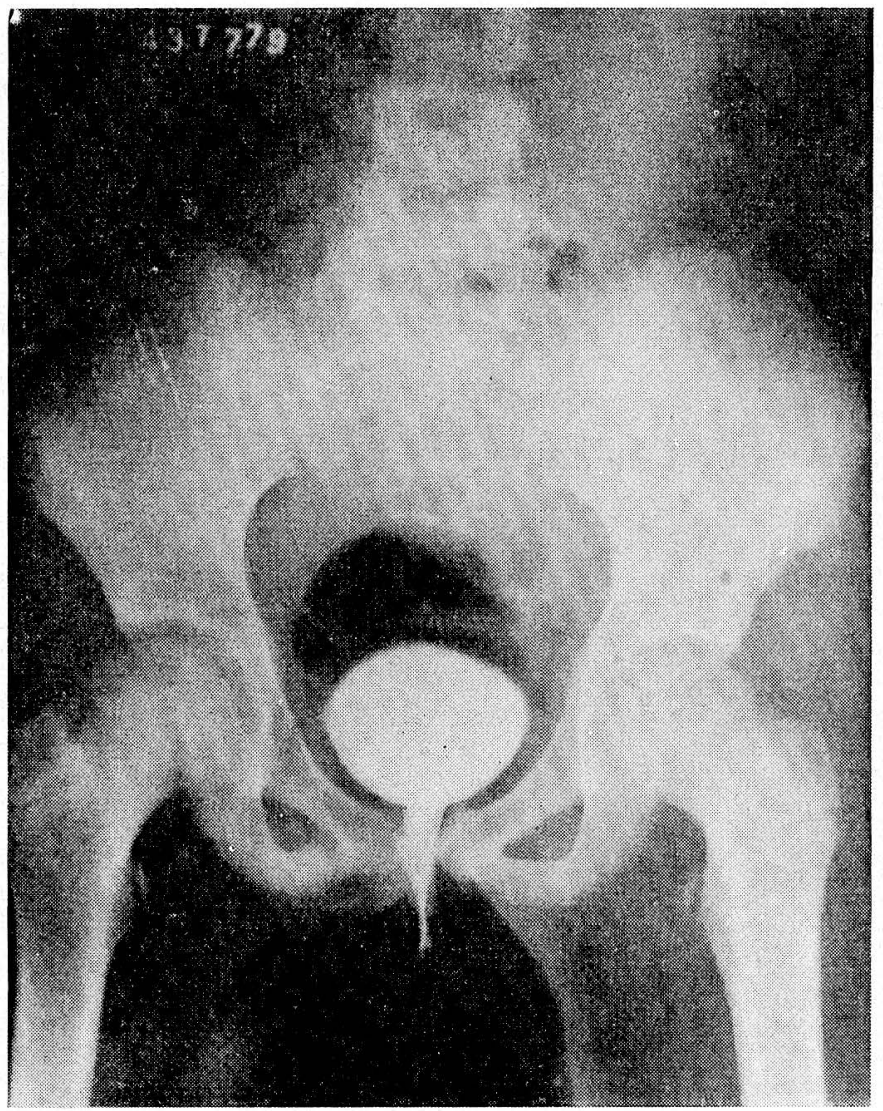

FIGURA Nọ 9 - Aspecto radiológico, operación para corregir dilatación congénita de la uretra, preoperatorio. (A.P.). 


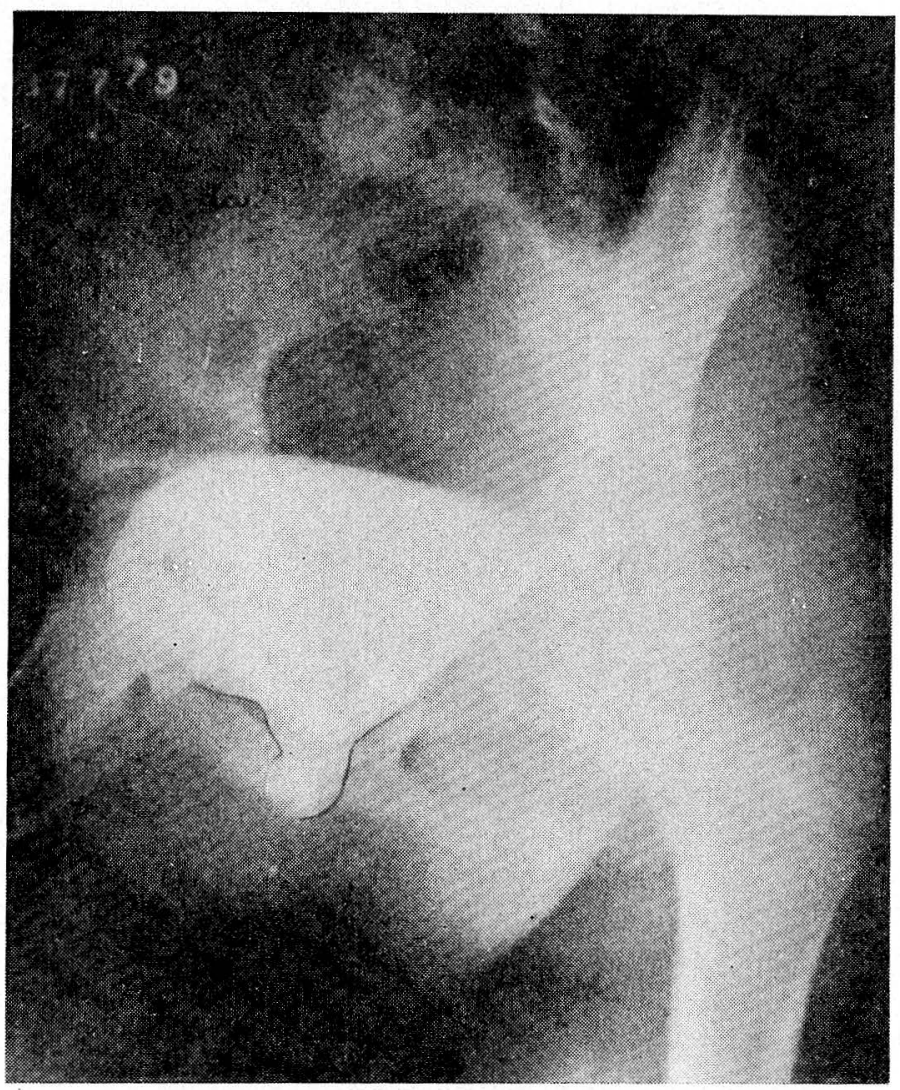

FIGURA $N^{0} 10$ - El caso anterior con una radiografía lateral. 


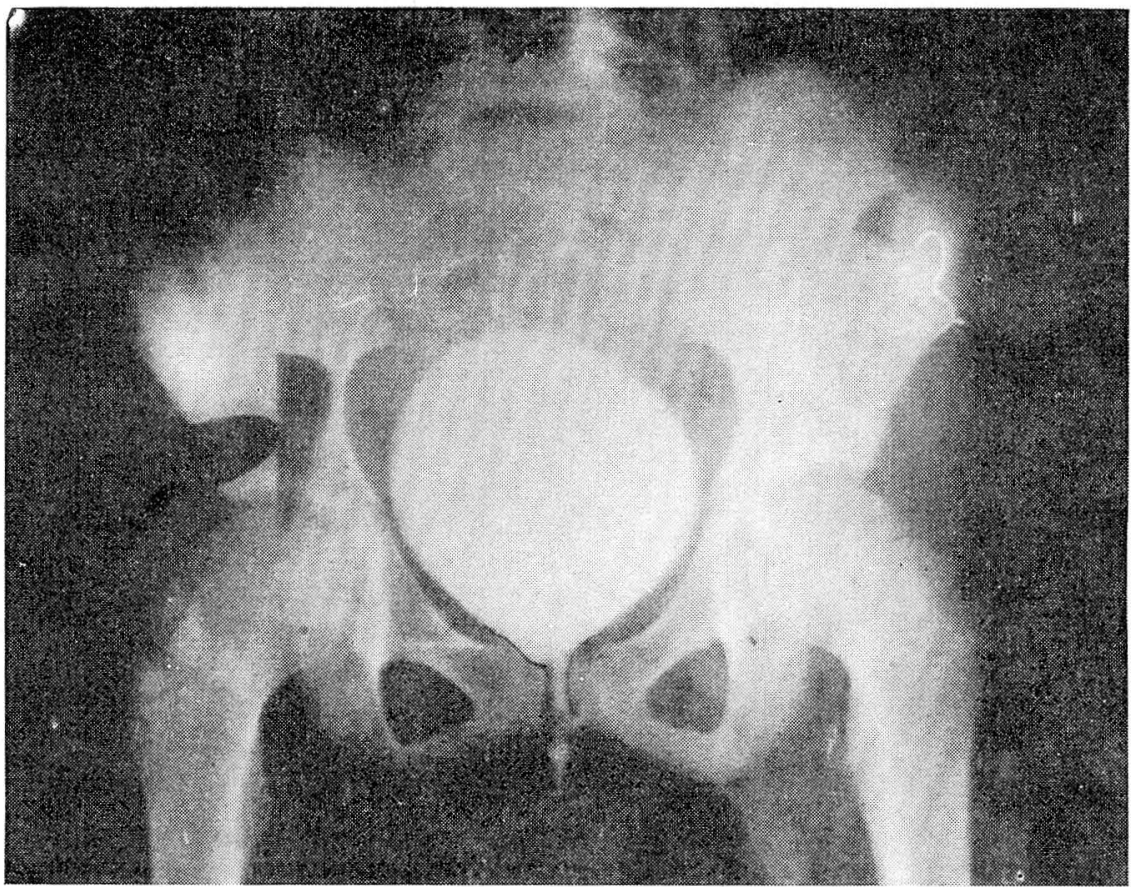

FIGURA $\mathrm{N}^{0} 11$ - Aspecto radiológico post-operatorio para corregir dilatación congénita de la uretra.

\section{Summary and Conclusions}

In agreement with the thesis presented and fully proven on urinary continency:

1. There is no sphincter but a sphincterial mechanism formed by a complex muscular mechanism that extends from the ureter, vesical trigon, the bladder neck and the urethra;

2. Urinary continency depends on four main mechanisms: tus.

a. Low intrinsical urinary appara-

b. Urogenital diaphragm.

c. Intra-abdominal pressure. d. Urethrovesical angle.

3. Any situation that fully or partially alter the above mechanisms shall produce urinary continency.

4. Thus, in agreement with the above, there are several types of urinary continency.

5. Each type of urinary continency has its modality for correction and, therefore, the diagnosis must be as exact as possible in order that the corresponding surgical treatment be applied to each case.

6. Certain surgical techniques are mentioned to correct such incontinency, without detailing the procedure. 


\section{AGRADECIMIENTOS A DOS DOCTORES :}

Dr. Arturo Rodríguez Soto - Instructor Asociado - Sección de Ginecología y Obstetricia Facultad de Medicina U.N.

Dr. Humberto Mouthon Yamal - Residente 4 Unidad de Urología - Facultad de Medicina U. N.

Por su colaboración en este trabajo.

\section{BIBLIOGRAFIA}

1 TANAGHO, MEYERS y SMITH: The Trigone: Anatomical and Physiolofcal consideration. 1. In relation to the Ureterovesical Junction. J. Urol. American. 100: 623, 1968.

2 TANAGHO, MEYER y SMITH: The Trigone: Anatomical and Physiological considerations. 2. In relation to the bladder neck. J. Urol. 100: 633, 1968.
Rev. Col. Obst. y Ginec.

3 TANAGHO, MEYER y SMITH: Mechanism of Urinary continence. 1. Embryologic, Anatomic and Pathologic considerations. J. Urol. 100 : 640, 1968.

4 BERNAL $H$. E.: La uretrovésico suspensión de Marshall Marcheti-Krantz en la corrección de la incontinencia urinaria de esfuerzo. Rev. Col. Obst. y Gin. XV: 159, 1964.

5 ENHORNING, G.: Simultaneous recording of intravesical and intra-urethral pressure. A study on urethral closure in normal and stress incontinent women. Acta Chir. Scand. suppl. $276,1961$.

6 ENHORNING, G., MILLER E. R. and HINMAN, F., Jr.: Urethral closure studied with cineroent-genography and simultaneous bladder urethra pressure recording. Surg., Gynec. \& Obst., 118: 507, 1964.

7 TANAGHO, E. A., MILLER, E. R., MEYERS, F. H. and CORBETT, B. K.: Observations on the dynamics of the bladder neck. Brit. J. Urol., 38: 72, 1966. 